\title{
CLOSED-LOOP TRANSIENT STABILITY EMERGENCY CONTROL
}

\author{
Damien Ernst* and Mania Pavella \\ University of Liège, Sart-Tilman B28, B - 4000 Liège, Belgium \\ ${ }^{*}$ Research Fellow, FNRS
}

\begin{abstract}
The question of transient stability control is revisited, various types of controls are identified, and a general approach to closed-loop emergency control is proposed. The focus is on feasibility aspects, general salient features and illustration of stabilization capabilities of an emergency control scheme relying on generation shedding.
\end{abstract}

Keywords: Transient stability; Emergency control; SIME.

\section{INTRODUCTION}

Power system transient sability control encompasses in general a twofold problem: severity assessment of an instability originating from the occurrence of a "dangerous" contingency, and choice of an action able to stabilize it. The control may be of the "preventive" type or of the "emergency" type.

Preventive control aims at answering the question "what to do" in order to stabilize the system post-fault operating condition if a (plausible) contingency would occur. Its design relies on stability simulations of contingency scenarios. More precisely, on-line preventive control aims at designing in a horizon of, say, 30 minutes ahead, means to stabilize the system if it were threatened by any of the plausible contingencies identified to be dangerous. The decision about whether to execute or postpone the resulting control action relies on engineering judgement.

Emergency control, on the other hand, aims at triggering a control action in real time, after a dangerous contingency has actually occurred. Note that this control action may be either designed in real time using real-time measurements or assessed in anticipation by means of off-line stability simulations. The latter case belongs to open-loop emergency control. In contrast, in closed-loop emergency control the action is designed and triggered in real time, after a disturbance has actually occurred, and the system continues being monitored and further controlled if necessary.

Conventional time-domain transient stability methods can hardly tackle preventive control, and are totally unable to deal with closed-loop emergency control. Hybrid methods seem better suited to such a problematic task. In particular, the method called SIME [1] has been able to solve properly preventive aspects (e.g., see [2], and the related references therein). More recently, the Emergency SIME was proposed for closed-loop emergency control $[3,4]$.

This contribution deals with the Emergency SIME in general, then with its illustration on the WSCC power system, where the control action is supposed to be generation shedding.
The presentation is organized as follows. Section 2 gives a digest of the SIME method in general. Section 3 deals with the Emergency SIME. Finally, the particular control of generation shedding is illustrated in Section 4.

\section{THE PREDICTIVE SIME}

\subsection{Principle}

In essence, SIME assesses the behavior of a power system in its post-fault configuration (after a disturbance inception and its clearance) in terms of a generalized one-machine-infinitebus (OMIB) transformation [5]. This OMIB equivalent results from the aggregation of the groups of "critical machines" and "non-critical machines" into two equivalent machines, further replaced by a one-machine equivalent. ${ }^{1}$

The identification of these two groups of machines is described below and portrayed in Fig. 1, drawn for the example of Section 4. The time-dependent parameters of the "generalized OMIB" (rotor angle and speed; mechanical and electrical powers), are computed from the parameters of the power system machines. In the Emergency SIME,these multimachine parameters are furnished by real-time measurements, and refreshed at a regular rate.

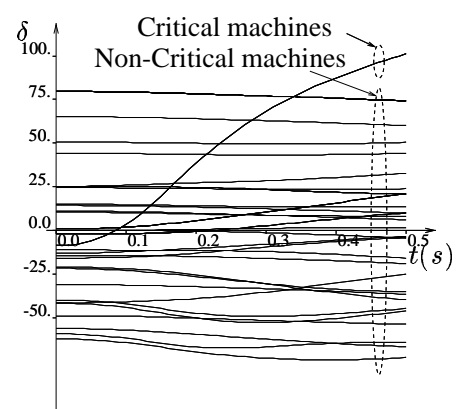

(a) Power system swing curves and critical machine identification

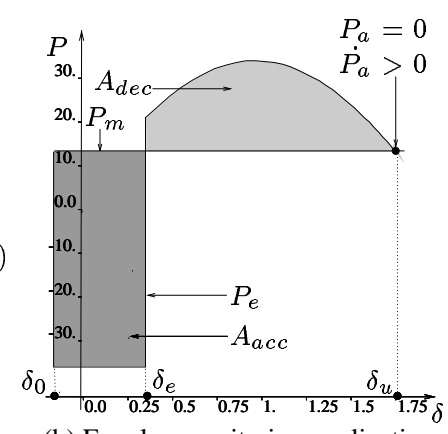

(b) Equal area criterion application to the OMIB equivalent system
Figure 1. Illustration of SIME's principle

The stability properties of the OMIB are inferred from the application of the equal-area criterion (EAC). This yields two essential pieces of information, namely, stability margins and critical machines. A stability margin expresses the imbalance between accelerating and decelerating areas of the OMIB $P-\delta$ plane, or, equivalently, of the $P_{a}-\delta$ plane (see Fig. 1b), where

\footnotetext{
${ }^{1}$ Note that this is a generalized version of the extended equal area criterion (EEAC) [6].
} 
$P_{a}=P_{m}$ (its mechanical power) $-P_{e}$ (its electrical power) .

\subsection{Predictive transient stability assessment}

Following a disturbance inception and its clearance, the Emergency SIME aims at predicting the system transient stability behavior and, if necessary, at deciding and triggering control actions early enough to prevent loss of synchronism. Further, it aims at continuing monitoring the system, in order to assess whether the control action has been sufficient or should be reinforced. The prediction relies on real-time measurements, acquired at regular time step, $t_{i}$ 's, and refreshed at the rate $\Delta t_{i}$. The procedure consists of the following tasks. .

(i) Predicting the OMIB structure: use a Taylor series expansion to predict (say, $100 \mathrm{~ms}$ ahead), the individual machines rotor angles; rank the machines according to their angles, identify the largest angular difference ("gap") between two successive machines and declare those above this gap to be the "candidate critical machines", those below the gap being the "candidate non-critical machines". Their aggregation provides the "candidate OMIB".

(ii) Predicting the $P_{a}-\delta$ curve: compute the parameters of this "candidate OMIB", and in particular its accelerating power and rotor angle, $P_{a}$ and $\delta$, for three successive data sets acquired at $t_{i}, t_{i}+\Delta t_{i}, t_{i}+2 \Delta t_{i}$. Write the equation

$$
P_{a}(\delta)=a \delta^{2}+b \delta+c
$$

for the three different times and solve for $a, b, c$.

(iii) Predicting instability: search for the solution of

$$
P_{a}\left(\delta_{u}\right)=a \delta_{u}^{2}+b \delta_{u}+c=\boldsymbol{\sigma}
$$

to determine whether the OMIB reaches the unstable conditions

$$
P_{a}\left(\delta_{u}\right)=\boldsymbol{\sigma}, \dot{P}_{a}\left(\delta_{u}\right)>\boldsymbol{\sigma} .
$$

If not, repeat steps (i) to (iii) using new measurements sets.

If yes, the candidate OMIB is the critical one, for which the method computes successively $[3,4]$

- the unstable angle, $\delta_{u}$

- the unstable margin

$$
\eta=-\int_{\delta_{i}}^{\delta_{u}} P_{a} d \delta-\frac{1}{2} M \omega_{i}^{2}
$$

- the time to instability

$$
t_{u}=t_{i}+\int_{\delta_{i}}^{\delta_{u}} \frac{d \delta}{\sqrt{\frac{2}{M} \int_{\delta_{i}}^{\delta}-P_{a} d \delta+\omega_{i}^{2}}}
$$

where $\delta_{i}$ stands for $\delta\left(t_{i}\right)$ and $\omega_{i}$ for $\omega\left(t_{i}\right)$.

(iv) Validity test. The validity test relies on the observation that under given operating and contingency conditions, the value of the (negative) margin should be constant, whatever the time step. Hence, the above computations should be repeated at successive $\Delta t_{i}$ 's until getting an (almost) constant margin value.
The method is based on real-time measurements acquired at regular time intervals and aims at controlling the system in less than, say, $500 \mathrm{~ms}$ after the contingency inception and its clearance.

- The prediction phase starts after detecting an anomaly (contingency occurrence) and its clearance by means of protective relays. Note that this prediction does not imply identification of the contingency (location, type, etc.).

- The prediction is possible thanks to the use of the OMIB transformation; predicting the behavior (accelerating power) of all of the system machines would have led to totally unreliable results.

- There may be a tradeoff between the above mentioned validation test and the time to instability: the shorter this time, the faster the corrective action should be taken. On the other hand, the shorter the time to instability, the earlier the instability phenomena appear.

Finally note that the above descriptions aim at giving a mere flavor of the method. Detailed developments may be found in $[3,4]$.

\section{EMERGENCY CONTROL}

\subsection{General principle}

Transient stability control relies on the following two propositions:

(i) the degree of instability of a multi-machine power system is measured by the OMIB margin;

(ii) stabilizing an unstable case consists of canceling out this margin, i.e. of increasing the decelerating area and/or decreasing the accelerating area in the OMIB $P-\delta$ plane (see Fig. 1b).

Broadly, this may be achieved either by:

- reducing the mechanical power of the OMIB. E.g., by using fast-valving, generator shedding, generator rescheduling, etc.;

- increasing the electrical power. E.g., by using braking resistors, DC links, thyristor controlled series compensators, and other FACTS.

Emergency control, in particular, aims at protecting large production sites, like hydro plants; it may call upon last resort actions, e.g., generation shedding.

The above principle is schematically organized in the clossed-loop control framework portrayed in Fig. 2. The core of this Emergency SIME consists of blocks 2 and 3, commented below in the particular case of generation shedding.

\subsection{Structure of the emergency control scheme}

Let us summarize the main objectives of the Emergency SIME discussed so far. On the basis of real-time measurements taken at the power plants, the method aims to [3]: 


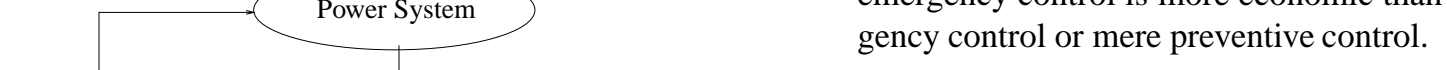

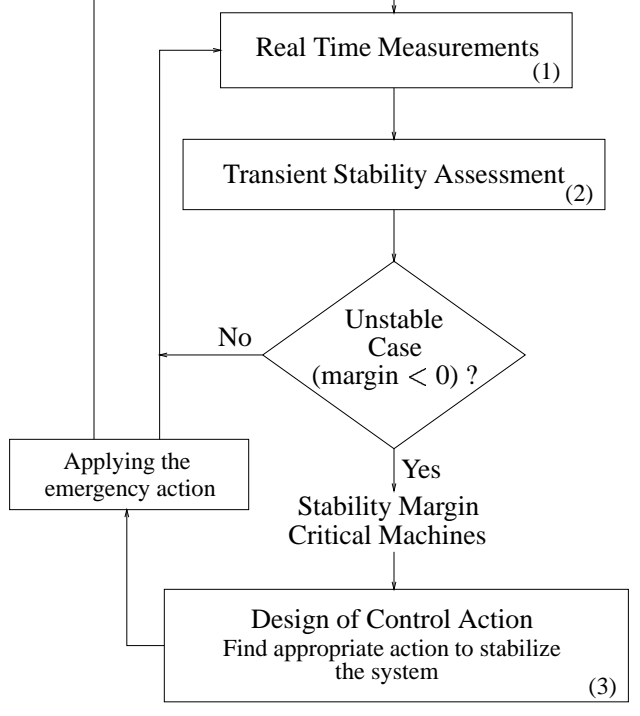

Figure 2. A general framework for closed-loop transient stability emergency control

- assess whether the system is stable or it is driven to instability; in the latter case;

- assess "how much" unstable the system is going to be; accordingly,

- assess "where" and "how much corrective action" to take (pre-assigned type of corrective action);

- to continue assessing whether the executed corrective action has been sufficient or whether to proceed further.

Block 2 of Fig. 2 covers the two first steps: prediction of (in)stability, and appraisal of the size of instability, in terms of margins and critical machines. Block 3 takes care of the design of control actions. For example, when generation shedding is of concern, the action consists of determining the number of generators to shed.

Further, the method sends the order of triggering the action, while continuing to monitor and control the system in closedloop until getting power system stabilization.

\subsection{Discussion}

- The prediction of the time to (reach) instability may influence the control decision (size of control; time to trigger it; etc).

- The hardware requirements of the emergency control scheme are phasor measurement devices placed at the main power plant stations and communication systems to transmit (centralize-decentralize) this information. These requirements seem to be within reach of today's technology [7].

- The emergency control relies on purely real-time measurements (actually a relatively small number of measurements). This frees the control from uncertainties about power system modeling, parameter values, operating condition, type and location of the contingency. Besides, such a closed-loop
These important advantages of emergency control make it a valuable complement to preventive control but certainly not a substitute. Indeed, it is at the junction of the two above types of control techniques that satisfactory solutions to particularly challenging operating problems could be found.

\section{ILLUSTRATION}

\subsection{Description}

The simulation reported in this section aims to show how the proposed scheme works in a practical case of generation shedding.

The considered network is the WSCC system; it was initially modelled with 29 machines, 179 buses and a total load of about $60,000 \mathrm{MW}$. However, most of the machines were in fact large equivalents; one of them has been split in 2 units, in order to be able to use finer assessment of generation shedding than that consisting of shedding a big unit.

The disturbance considered here is a three-phase short-circuit followed by the tripping of one line to clear the fault. The location of the fault was chosen at random among a number of "dangerous" scenarios, for which the system is driven to loss of synchronism. Note however that to get "dangerous" scenarios the considered fault clearing time was quite large $(150 \mathrm{~ms})$; with modern circuit breakers it is possible to get clearing times of less than $50 \mathrm{~ms}$ ( 3 cycles).

The overall delay for the generation shedding is considered equal to $150 \mathrm{~ms}: 50 \mathrm{~ms}$ to centralize all the measurements, $50 \mathrm{~ms}$ to send the corrective action order, and $50 \mathrm{~ms}$ to effectively shed the generator(s).

For want of real-time measurements, the simulations reported below have been run with SIME coupled with the ETMSP transient stability program [8].

\subsection{Three-phase short-circuit at bus MOHAVE 500}

A three-phase short-circuit is applied at bus MOHAVE 500 during $150 \mathrm{~ms}$. The fault is cleared by opening the line LUGO 500; MOHAVE 500.

\subsubsection{Simulation without emergency control}

To get a better understanding of the phenomena involved, let us first explore how the system behaves in the absence of emergency control tools. In this case, the scenario is unstable, and the time to instabilitiy is found to be of $490 \mathrm{~ms}$ after the fault inception. The number of critical machines, i.e. the machines responsible for the loss of synchronism of the system, is two, namely: MOHAV1CC22.0;1 and MOHAV1CC22.0;2; their initial power is $843 \mathrm{MW}$. Since these machines have exactly the same model and are connected to the same bus, their swing curves are identical.

The normalized unstable margin is negative and equal to $-2.8(\mathrm{rad} / \mathrm{s})^{2}$. Fig. 1a shows the swing curves of the 30 system machines. In the figure, the swing curves of the critical machines coincide and are represented by the most ad- 
ber among these machines necessary to stabilize the system.

Fig. 1b represents the mechanical and electrical power of the OMIB in the power-angle plane. The difference between the decelerating and accelerating areas gives the value of the margin. The unstable angle $\delta_{u}$ is equal to $1.75 \mathrm{rad}$; it corresponds to a speed, $\omega_{u}$ of $2.36 \mathrm{rad} / \mathrm{s}$.

\subsubsection{Simulation with emergency control.}

The above contingency is here stabilized using the emergency SIME and following the steps described in Sections 2 and 3 .

Thus, to determine if the system is driven to instability, the margin is predicted by SIME at each time step of the ETMSP program, by extrapolating the curve of the accelerating power of the OMIB in the power-angle plane. If the margin is found to be negative, a corrective action must be designed and triggered, in order to stabilize the system. The design of this corrective action, i.e. which and how many machines to shed, is made by using a similar extrapolation. To a corrective action is associated a new margin, refreshed at each time a new set of measurements is acquired (here, at each time step of the program). A positive value of this new margin means that the action has been sufficient. Otherwise, an additional corrective action must be taken in order to avoid loss of synchronism.

Table 1 reports on simulation results. The rate $\Delta t_{i}$ between two successive time samples is equal to $10 \mathrm{~ms}$.

Table 1.

\begin{tabular}{|c|c|c|c|}
\hline 1 & 2 & 3 & 4 \\
\hline $\begin{array}{c}\text { Time } \\
(\mathbf{m s})\end{array}$ & $\begin{array}{c}\text { Margin before } \\
\text { corrective action } \\
\left(\mathbf{r a d} / \mathbf{s}^{2}\right)\end{array}$ & $\begin{array}{c}\text { Time to } \\
\text { instability } \\
(\mathbf{m s})\end{array}$ & $\begin{array}{c}\text { Margin after } \\
\text { corrective action } \\
\left(\mathbf{r a d} / \mathbf{s}^{2}\right)\end{array}$ \\
\hline 290 & -3.54 & 458 & $/$ \\
300 & -3.37 & 463 & 0.874 \\
310 & -3.35 & 464 & 0.903 \\
320 & -3.31 & 465 & 1.105 \\
330 & -3.27 & 466 & 1.208 \\
340 & -3.21 & 468 & 1.322 \\
350 & -3.16 & 469 & 1.487 \\
360 & -3.11 & 471 & 1.748 \\
370 & -3.05 & 473 & 1.902 \\
380 & -2.99 & 475 & 2.142 \\
390 & -2.97 & 475 & 2.025 \\
400 & -2.94 & 476 & 2.330 \\
410 & -2.90 & 478 & 2.541 \\
420 & -2.87 & 479 & 2.676 \\
430 & -2.83 & 481 & 2.827 \\
440 & -2.81 & 480 & 2.835 \\
\hline
\end{tabular}

The content of the table is as follows.

Column \# 1 : time of the last set of measurements

Column \# 2: predicted margin of the uncontrolled system

Column \# 3 : predicted time to instability

Column \# 4 : predicted margin of the controlled system.

Before $290 \mathrm{~ms}$ the predicted margin is positive, which corresponds to a stable system. But at $290 \mathrm{~ms}$ the margin becomes negative; the corresponding time to instability is of $458 \mathrm{~ms}$. is decided, consisting of shedding one critical machine. This action will be triggered $150 \mathrm{~ms}$ later. The system continues being monitored at each acquisition of a new set of measurements by refreshing the values of the margins. Observe that the predicted margin of the controlled system increases, suggesting that the corrective action was sufficient.

Table 1 shows that shedding one generator increases the value of the margin, which becomes positive, suggesting that the system has been stabilized (see column 4). Fig. 3a shows that shedding one critical machine increases the decelerating area, as predicted. Note that the unstable angle shown in Fig. 3a, which is approximately 2 radians, is never reached thanks to the fact that the additional decelerating area is sufficient to bring the system back to synchronism. A return angle appears before the system loses synchronism.

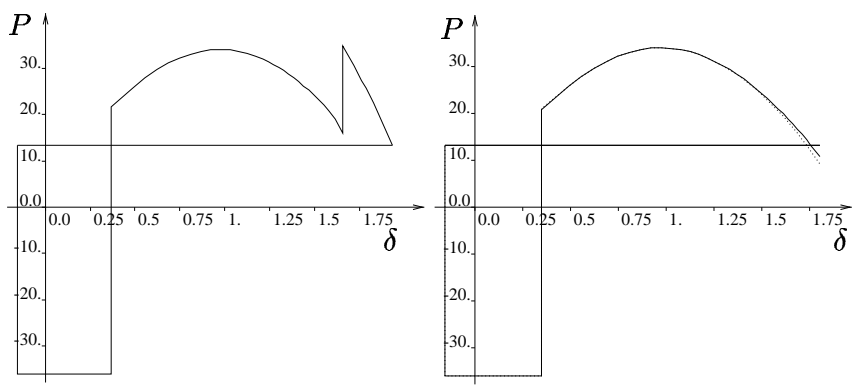

(a) Shedding of one generator (b) Accurancy of the extrapolation

Figure 3. $P-\delta$ planes

\section{Remark}

The accuracy of the method relies on a good prediction of the accelerating power of the OMIB in the power-angle plane. To illustrate it, consider Fig. 3b. The solid line portrays the exact curve, the same as shown in Fig. 1b. Table 1 shows that the predicted value of the margin at time $300 \mathrm{~ms}$ is smaller than the exact values $\left(-3.37(\mathrm{rad} / \mathrm{s})^{2}\right.$ as opposed to $\left.-2.8(\mathrm{rad} / \mathrm{s})^{2}\right)$. This is because the predicted curve is below the real one so that the computed decelerating area is smaller that the real one; this leads to an overestimation of the degree of the system instability.

\section{CONCLUSION}

Direct methods gradually changed their scope during more than three decades of development. Indeed, one of their primary objectives was to speed transient stability computations and to get simulations "faster than real time". Today, this objective is well within reach of "brute force" time-domain programs, thanks to the fantastic progress of computer performances.

Meanwhile, the secure operation of modern power systems has created needs impossible to meet by pure time-domain methods. Transient stability control is a good example. At the same time, pragmatic approaches resulting from the hybridization of direct methods became able to meet such stringent requirements. Also in parallel, progress of technology has made possible hardware requirements concerning data acquisition and communication. The Emergency SIME presented 
hardware advances. It is anticipated that the deregulation of the electric industry will contribute to speed up the effective implementation of such modern tools.

\section{REFERENCES}

[1] "SIME: A hybrid Approach to Fast Transient Stability Assessment and Contingency Selection", Y. Zhang, L. Wehenkel, P. Rousseaux and M. Pavella. EPES, Vol.19, No.3, 1997, pp. 195208.

[2] "Transient Stability-Constrained Maximum Allowable Transfer", L. Bettiol, L. Wehenkel and M. Pavella. IEEE Trans. on Power systems, Vol.14, No.2, May 1999, pp. 654-659.

[3] "A Method for Real-Time Transient Stability Emergency Control", Y. Zhang, L. Wehenkel and M. Pavella. Proc. of CPSPP'97, IFAC/CIGRE Symp. on Control of Power Systems and Power Plants, August 1997, Beijing, China, pp. 673-678.

[4] "Real Time Transient Stability Emergency Control of the SouthSoutheast Brazilian System", D. Ernst, A. Bettiol, Y. Zhang, L. Wehenkel and M. Pavella. SEPOPE, Salvador, Brazil, May 1998, (Invited paper, IP044; 9 pages).

[5] "Generalized One-Machine Equivalents in Transient Stability Studies", M. Pavella. PES Letters, IEEE Power Engineering Review, Vol.18, No.1, January 1998, pp. 50-52. See also Ref. [3] therein.

[6] "A simple direct method for fast transient stability assessment of large power systems", Y. Xue, Th. Van Cutsem and M. RibbensPavella (1998). IEEE Trans. PWRS, 3: pp. 400-412.

[7] "Synchronized Phasor Measurements in Power Systems", A.G. Phadke. IEEE Computer Applications in Power, Vol.6, No.2, April 1993, pp. 10-15.

[8] "Standard Test Cases for Dynamic Security Assessment", Electric Power Research Institute. Final EPRI report No. EPRI TR-105885, Project 3103-02-03, December, 1995.

[9] WSCC reports on July 2, 1996 and August 10, 1996 outages available at http://www.wscc.com.

Acknowledgements. One of us (Damien Ernst) is grateful to Professor Arun Phadke for his kind hospitality during his stay at Virginia Tech, and for generously providing advice and guidance. 